\title{
A Relativistic Algorithm with Isotropic Coordinates
}

\author{
S. A. Ngubelanga and S. D. Maharaj \\ Astrophysics and Cosmology Research Unit, School of Mathematics, Statistics and Computer Science, \\ University of KwaZulu-Natal, Private Bag X54001, Durban 4000, South Africa \\ Correspondence should be addressed to S. D. Maharaj; maharaj@ukzn.ac.za
}

Received 9 June 2013; Accepted 18 November 2013

Academic Editor: Stephen C. Anco

Copyright (C) 2013 S. A. Ngubelanga and S. D. Maharaj. This is an open access article distributed under the Creative Commons Attribution License, which permits unrestricted use, distribution, and reproduction in any medium, provided the original work is properly cited.

\begin{abstract}
We study spherically symmetric spacetimes for matter distributions with isotropic pressures. We generate new exact solutions to the Einstein field equations which also contain isotropic pressures. We develop an algorithm that produces a new solution if a particular solution is known. The algorithm leads to a nonlinear Bernoulli equation which can be integrated in terms of arbitrary functions. We use a conformally flat metric to show that the integrals may be expressed in terms of elementary functions. It is important to note that we utilise isotropic coordinates unlike other treatments.
\end{abstract}

\section{Introduction}

We consider the interior of static perfect fluid spheres in general relativity with isotropic pressures. The predictions of general relativity have been shown to be consistent with observational data in relativistic astrophysics and cosmology. For a discussion of the physical features of a gravitating model, we require an exact solution to the Einstein field equations. Exact solutions are crucial in the description of dense relativistic astrophysical problems. Many solutions have been found in the past. For some comprehensive lists of known solutions to the field equations, refer to Delgaty and Lake [1], Finch and Skea [2], and Stephani et al. [3]. Many of these solutions are not physically reasonable. For physical reasonableness, we require that the gravitational potentials and matter variables are regular and well behaved, causality of the spacetime manifold is maintained and values for physical quantities, for example, the mass of a dense star, are consistent with observation.

Solutions have been found in the past by making assumptions on the gravitational potentials, matter distribution, or imposing an equation of state. These particular approaches do yield models which have interesting properties. However in principle, it would be desirable to have a general method that produces exact solutions in a systematic manner. Some systematic methods generated in the past are those of Rahman and Visser [4], Lake [5], Martin and Visser [6], Boonserm et al. [7], Herrera et al. [8], Chaisi and Maharaj [9], and Maharaj and Chaisi [10]. In general relativity, we have the freedom of using any well-defined coordinate system. The references mentioned above mainly use canonical coordinates. The use of isotropic coordinates may provide new insights and possibly lead to new solutions. This is the approach that we follow in this paper. We generate a new algorithm producing a new solution, to Einstein field equations in isotropic coordinates. From a given solution we can find a new solution with isotropic pressures.

The objective of this paper is to find new classes of exact solutions of the Einstein field equations with an uncharged isotropic matter distribution from a given seed metric. In Section 2, we derive the Einstein field equations for neutral perfect fluids in static spherically symmetric spacetime. We introduce new variables due to Kustaanheimo and Qvist [11] to rewrite the field equations and the condition of pressure isotropy in equivalent forms. In Section 3, we introduce our algorithm and the master nonlinear second order differential equation containing two arbitrary functions, that has to be solved. In Section 4, we present new classes of exact solutions in terms of the arbitrary functions. In Section 5, we give an example for a conformally flat metric showing that the integrals generated in Section 4 may be explicitly evaluated. In Section 6, we summarise the results obtained in this paper. 


\section{The Model}

We are modelling the interior of a dense relativistic star in strong gravitational fields. The line element of the interior spacetime, with isotropic coordinates, has the following form:

$$
d s^{2}=-A^{2}(r) d t^{2}+B^{2}(r)\left[d r^{2}+r^{2}\left(d \theta^{2}+\sin ^{2} \theta d \phi^{2}\right)\right],
$$

where $A(r)$ and $B(r)$ are arbitrary functions representing the gravitational potentials. Relativistic compact objects such as neutron stars in astrophysics are described by this line element. The energy momentum tensor for the interior of the star has the form of a perfect fluid

$$
T^{a b}=(\rho+p) u^{a} u^{b}+p g^{a b},
$$

where $\rho$ is the energy density and $p$ is the isotropic pressure. These quantities are measured relative to a timelike unit fourvelocity $u^{a}\left(u^{a} u_{a}=-1\right)$.

The Einstein field equations for (1) and (2) have the form

$$
\begin{gathered}
\rho=-\frac{1}{B^{2}}\left[2 \frac{B^{\prime \prime}}{B}-\frac{B^{\prime}}{B}\left(\frac{B^{\prime}}{B}-\frac{4}{r}\right)\right] \\
p=2 \frac{A^{\prime}}{A}\left(\frac{B^{\prime}}{B^{3}}+\frac{1}{r} \frac{1}{B^{2}}\right)+\frac{B^{\prime}}{B^{3}}\left(\frac{B^{\prime}}{B}+\frac{2}{r}\right) \\
p=\frac{1}{B^{2}}\left(\frac{A^{\prime \prime}}{A}+\frac{1}{r} \frac{A^{\prime}}{A}\right)+\frac{1}{B^{2}}\left[\frac{B^{\prime \prime}}{B}-\frac{B^{\prime}}{B}\left(\frac{B^{\prime}}{B}-\frac{1}{r}\right)\right]
\end{gathered}
$$

in isotropic coordinates. Primes denote differentiation with respect to the radial coordinate $r$. On equating (3b) and (3c), we obtain the condition of pressure isotropy which has the form

$$
\frac{A^{\prime \prime}}{A}+\frac{B^{\prime \prime}}{B}=\left(\frac{A^{\prime}}{A}+\frac{B^{\prime}}{B}\right)\left(2 \frac{B^{\prime}}{B}+\frac{1}{r}\right)
$$

This is the master equation which has to be integrated to produce an exact solution to the field equations.

It is possible to write the system (3a)-(3c) in an equivalent form by introducing new variables. We utilize a transformation that has proven to be helpful in relativistic stellar physics. We introduce the new variables

$$
x \equiv r^{2}, \quad L \equiv B^{-1}, \quad G \equiv L A .
$$

The above transformation was first suggested by Kustaanheimo and Qvist [11]. On applying transformation (5) in the field equations of (3a)-(3c), we obtain the equivalent system

$$
\begin{gathered}
\rho=4\left[2 x L L_{x x}-3\left(x L_{x}-L\right) L_{x}\right], \\
p=4 L\left(L-2 x L_{x}\right) \frac{G_{x}}{G}-4\left(2 L-3 x L_{x}\right) L_{x}, \\
p=4 x L^{2} \frac{G_{x x}}{G}+4 L\left(L-2 x L_{x}\right) \frac{G_{x}}{G} \\
-4\left(2 L-3 x L_{x}\right) L_{x}-8 x L L_{x x} .
\end{gathered}
$$

We note that (6a)-(6c) are highly nonlinear in both $L$ and $G$. In this system, there are three independent equations and four unknowns $\rho, p, L$, and $G$. So we need to choose the functional form for $L$ or $G$ in order to integrate and obtain an exact solution. The value of the transformation (5) is highlighted in the reduction of the condition of pressure isotropy. On equating (6b) and (6c), we get

$$
L G_{x x}=2 G L_{x x}
$$

which is the new condition of pressure isotropy which has a simpler compact form.

\section{The Algorithm}

It is possible to find new solutions to the Einstein's equations from a given seed metric. Examples of this process are given in the treatments of Chaisi and Maharaj [9] and Maharaj and Chaisi [10]. They found new models, with anisotropic pressures, from a given seed isotropic metric in Schwarzschild coordinates. Our intention is to find new models, with isotropic pressures, from a given solution in terms of the isotropic line element (1).

We can provide some new classes of exact solutions to the Einstein field equations by generating a new algorithm that produces a model from a given solution. We assume a known solution of the form $(\bar{L}, \bar{G})$, so that

$$
\bar{L} \bar{G}_{x x}=2 \bar{G} \bar{L}_{x x}
$$

holds. We seek a new solution $(L, G)$ given by

$$
L=\bar{L} e^{g(x)}, \quad G=\bar{G} e^{f(x)},
$$

where $f(x)$ and $g(x)$ are arbitrary functions. On substituting (9) into (7), we obtain

$$
\begin{aligned}
& \left(\bar{L} \bar{G}_{x x}-2 \bar{G} \bar{L}_{x x}\right)+2\left(\bar{L} \bar{G}_{x} f_{x}-2 \bar{G} \bar{L}_{x} g_{x}\right) \\
& +\bar{L} \bar{G}\left(f_{x x}-2 g_{x x}\right)+\bar{L} \bar{G}\left(f_{x}^{2}-2 g_{x}^{2}\right)=0,
\end{aligned}
$$

which is given in terms of two arbitrary functions $f(x)$ and $g(x)$. Then realizing that $(\bar{L}, \bar{G})$ is a solution of (7) and using (8), we obtain the reduced result

$$
\left(f_{x x}-2 g_{x x}\right)+2\left(\frac{\bar{G}_{x}}{\bar{G}} f_{x}-2 \frac{\bar{L}_{x}}{\bar{L}} g_{x}\right)+\left(f_{x}^{2}-2 g_{x}^{2}\right)=0 .
$$

We need to demonstrate the existence of functions $f(x)$ and $g(x)$ that satisfy (11). In general, it is difficult to integrate (11), since it is given in terms of two arbitrary functions which are nonlinear.

\section{New Solutions}

We consider several cases of (11) for which we have been able to complete the integration. 
4.1. $g(x)$ Is Specified. We can integrate (11) if $g(x)$ is specified. As a simple example, we take $g(x)=1$. Then (11) becomes

$$
f_{x x}+2 \frac{\bar{G}_{x}}{\bar{G}} f_{x}+f_{x}^{2}=0,
$$

which is nonlinear in $f$. This is a first order Bernoulli equation in $f_{x}$. We can rewrite (12) in the form

$$
\left(\frac{1}{f_{x}}\right)_{x}-2\left(\frac{\bar{G}_{x}}{\bar{G}}\right)\left(\frac{1}{f_{x}}\right)=1 .
$$

It is possible to integrate (13) since it is linear in $1 / f_{x}$ to obtain

$$
f_{x}=\bar{G}^{-2}\left(\int \bar{G}^{-2} d x+c_{1}\right)^{-1} \text {. }
$$

We can formally integrate (14) to obtain the function $f(x)$ as

$$
f(x)=\int\left[\bar{G}^{-2}\left(\int \bar{G}^{-2} d x+c_{1}\right)^{-1}\right] d x+c_{2},
$$

where $c_{1}$ and $c_{2}$ are arbitrary constants.

Then the new solution to (7) has the form

$$
\begin{gathered}
L=\bar{L}, \\
G=\bar{G} \exp \left(\int\left[\bar{G}^{-2}\left(\int \bar{G}^{-2} d x+c_{1}\right)^{-1}\right] d x+c_{2}\right) .
\end{gathered}
$$

Therefore we have shown that if a solution $(\bar{L}, \bar{G})$ to the field equations is known, then a new solution $(L, G)$ is given by (16a) and (16b).

4.2. $f(x)$ Is Specified. We can also integrate (11) if $f(x)$ is specified. As another simple example, we take $f(x)=1$. Then (11) becomes

$$
g_{x x}+2 \frac{\bar{L}_{x}}{\bar{L}} g_{x}+g_{x}^{2}=0,
$$

which is nonlinear in $g$. This is a first order Bernoulli equation in $g_{x}$. The differential equation (17) has a form similar to (12) in Section 4.1. Following the same procedure, we obtain

$$
g(x)=\int\left[\bar{L}^{-2}\left(\int \bar{L}^{-2} d x+c_{1}\right)^{-1}\right] d x+c_{2},
$$

where $c_{1}$ and $c_{2}$ are arbitrary constants.

Then another new solution to (7) is given by

$$
\begin{gathered}
G=\bar{G} \\
L=\bar{L} \exp \left(\int\left[\bar{L}^{-2}\left(\int \bar{L}^{-2} d x+c_{1}\right)^{-1}\right] d x+c_{2}\right) .
\end{gathered}
$$

Therefore we have determined that if a solution $(\bar{L}, \bar{G})$ to the field equations is known, then a new solution $(L, G)$ is given by (19a) and (19b). Note that the solution of (19a) and (19b) is different from that of (16a) and (16b).
4.3. $g(x)=\alpha f(x)$. We can integrate (11) if a relationship between the functions $f(x)$ and $g(x)$ exists. We illustrate this feature by assuming that

$$
g(x)=\alpha f(x)
$$

where $\alpha$ is an arbitrary constant. Then (11) becomes

$$
f_{x x}+\frac{2}{1-2 \alpha}\left(\frac{\bar{G}_{x}}{\bar{G}}-2 \alpha \frac{\bar{L}_{x}}{\bar{L}}\right) f_{x}+\left(\frac{1-2 \alpha^{2}}{1-2 \alpha}\right) f_{x}^{2}=0 \text {, }
$$

which is a first order Bernoulli equation in $f_{x}$. For convenience, we let

$$
\Theta=\left(\frac{1-2 \alpha^{2}}{1-2 \alpha}\right), \quad \eta=\frac{2}{1-2 \alpha}, \quad \alpha \neq \frac{1}{2},
$$

so that we can write (21) as

$$
\left(\frac{1}{f_{x}}\right)_{x}-\eta\left(\frac{\bar{G}_{x}}{\bar{G}}-2 \alpha \frac{\bar{L}_{x}}{\bar{L}}\right)\left(\frac{1}{f_{x}}\right)=\Theta,
$$

which is linear in $1 / f_{x}$. We integrate $(23)$ to obtain

$$
f_{x}=\left(\frac{\bar{L}^{2 \alpha}}{\bar{G}}\right)^{\eta}\left[\Theta \int\left(\frac{\bar{L}^{2 \alpha}}{\bar{G}}\right)^{\eta} d x+c_{1}\right]^{-1} .
$$

We now formally integrate (24) to obtain

$$
f(x)=\int\left(\left(\frac{\bar{L}^{2 \alpha}}{\bar{G}}\right)^{\eta}\left[\Theta \int\left(\frac{\bar{L}^{2 \alpha}}{\bar{G}}\right)^{\eta} d x+c_{1}\right]^{-1}\right) d x+c_{2}
$$

where $c_{1}$ and $c_{2}$ are constants.

We now have a new solution of (7) given by

$$
\begin{aligned}
L=\bar{L} \exp \alpha & {\left[\int\left(\frac{\bar{L}^{2 \alpha}}{\bar{G}}\right)^{\eta}\right.} \\
& \left.\left.\times\left[\Theta \int\left(\frac{\bar{L}^{2 \alpha}}{\bar{G}}\right)^{\eta} d x+c_{1}\right]^{-1}\right) d x+c_{2}\right],
\end{aligned}
$$

$$
\begin{aligned}
G=\bar{G} \exp \left[\int\left(\frac{\bar{L}^{2 \alpha}}{\bar{G}}\right)^{\eta}\right. \\
\left.\left.\qquad\left[\Theta \int\left(\frac{\bar{L}^{2 \alpha}}{\bar{G}}\right)^{\eta} d x+c_{1}\right]^{-1}\right) d x+c_{2}\right],
\end{aligned}
$$

where $\Theta$ and $\eta$ are given in (22). Therefore we have demonstrated that if a solution $(\bar{L}, \bar{G})$ to the field equations is 
specified, then a new solution $(L, G)$ is provided by (26a) and (26b).

Some special cases related to (26a) and (26b) should be pointed out. These relate to $\alpha=1, \pm 1 / \sqrt{2}, 1 / 2$. We consider each in turn.

Case $i(\alpha=1)$. With $\alpha=1$, we find that (26a) and (26b) become

$$
\begin{aligned}
& L=\bar{L} \exp \left(\int\left[\frac{\bar{G}^{2}}{\bar{L}^{4}}\left(\int \frac{\bar{G}^{2}}{\bar{L}^{4}} d x+c_{1}\right)^{-1}\right] d x+c_{2}\right), \\
& G=\bar{G} \exp \left(\int\left[\frac{\bar{G}^{2}}{\bar{L}^{4}}\left(\int \frac{\bar{G}^{2}}{\bar{L}^{4}} d x+c_{1}\right)^{-1}\right] d x+c_{2}\right),
\end{aligned}
$$

which are a simple form.

Case ii $(\alpha= \pm 1 / \sqrt{2})$. If we set $\alpha= \pm 1 / \sqrt{2}$, then (26a) and (26b) become

$$
\begin{gathered}
L=\bar{L} \exp \left[ \pm \frac{1}{\sqrt{2}}\left(c_{1} \int\left(\frac{\bar{L}^{ \pm \sqrt{2}}}{\bar{G}}\right)^{2 /(1-( \pm \sqrt{2}))} d x+c_{2}\right)\right] \\
G=\bar{G} \exp \left[c_{1} \int\left(\frac{\bar{L}^{ \pm \sqrt{2}}}{\bar{G}}\right)^{2 /(1-( \pm \sqrt{2}))} d x+c_{2}\right]
\end{gathered}
$$

which are another simple case.

Case iii ( $\alpha=1 / 2)$. If $\alpha=1 / 2$, then (26a) and (26b) are not valid. For this case, (11) becomes

$$
f_{x}\left[f_{x}+4\left(\frac{\bar{G}_{x}}{\bar{G}}-\frac{\bar{L}_{x}}{\bar{L}}\right)\right]=0 .
$$

When $f$ is constant, then $g$ is also constant by (20); then, (7) does not produce a new solution because of (9). When $f$ is not constant, then we can integrate (29) to produce the solution

$$
\begin{aligned}
& L=K \frac{\bar{L}^{3}}{\bar{G}^{2}}, \\
& G=K \frac{\bar{L}^{4}}{\bar{G}^{3}},
\end{aligned}
$$

where $K$ is a constant. Thus $\alpha=1 / 2$ generates another new solution $(L, G)$ to (11).

\section{Example}

We show by means of a specific example that the integrals generated in Section 4 may be evaluated to produce a new exact solution to the field equations in terms of elementary functions. In our example, we choose

$$
\begin{aligned}
& \bar{L}=b+a x, \\
& \bar{G}=1+c x .
\end{aligned}
$$

Then the corresponding line element is given by

$$
\begin{aligned}
d s^{2}= & -\left(\frac{1+c r^{2}}{b+a r^{2}}\right)^{2} d t^{2} \\
& +\left(\frac{1}{b+a r^{2}}\right)^{2}\left(d r^{2}+r^{2}\left(d \theta^{2}+\sin ^{2} \theta d \phi^{2}\right)\right),
\end{aligned}
$$

which is conformally flat. The energy density for the metric (32) is constant, so that we have the Schwarzschild interior solution in isotropic coordinates.

Conformally flat metrics are important in gravitational physics in a general relativistic setting. They arise, for instance, in the gravitational collapse of a radiating star, as shown in the treatments of Herrera et al. [12], Maharaj and Govender [13], Misthry et al. [14], and Abebe et al. [15]. For the choice of (31a) and (31b), we find that (27a) and (27b) become

$$
\begin{aligned}
L=(b+a x) \exp \left(\int\right. & \frac{(1+c x)^{2}}{(b+a x)^{4}} \\
& \left.\left.\times\left(\int \frac{(1+c x)^{2}}{(b+a x)^{4}} d x+c_{1}\right)^{-1}\right] d x+c_{2}\right), \\
G=(1+c x) \exp \left(\int\right. & {\left[\frac{(1+c x)^{2}}{(b+a x)^{4}}\right.} \\
& \left.\left.\times\left(\int \frac{(1+c x)^{2}}{(b+a x)^{4}} d x+c_{1}\right)^{-1}\right] d x+c_{2}\right) .
\end{aligned}
$$

The integrals in (33a) and (33b) can be evaluated and we obtain

$$
\begin{aligned}
& L=\frac{1}{(b+a x)^{2}} U(x), \\
& G=\frac{(1+c x)}{(b+a x)^{3}} U(x),
\end{aligned}
$$

where $c_{1}=0$ and $c_{2}=1$ and we have set

$$
U(x)=b^{2} c^{2}+a b c(1+3 c x)+a^{2}\left(1+3 c x+3 c^{2} x^{2}\right) .
$$

Thus the known solution $(\bar{L}, \bar{G})$ in (31a) and (31b) produces a new solution $(L, G)$ in (34a) and (34b). The line element for the new solution has the form

$$
\begin{aligned}
d s^{2}= & -\left(\frac{1+c r^{2}}{b+a r^{2}}\right)^{2} d t^{2}+\left(\frac{\left(b+a r^{2}\right)^{2}}{U(r)}\right)^{2} \\
& \times\left(d r^{2}+r^{2}\left(d \theta^{2}+\sin ^{2} \theta d \phi^{2}\right)\right),
\end{aligned}
$$

where $U(r)$ is given by (35). Thus our algorithm has produced a new (not conformally flat) solution to the Einstein's field equations. This has been generated from a seed conformally flat model. 


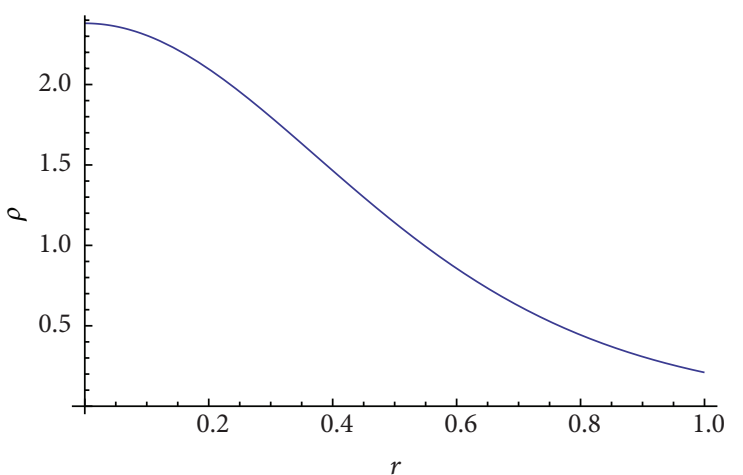

FIGURE 1: Energy density $\rho$.

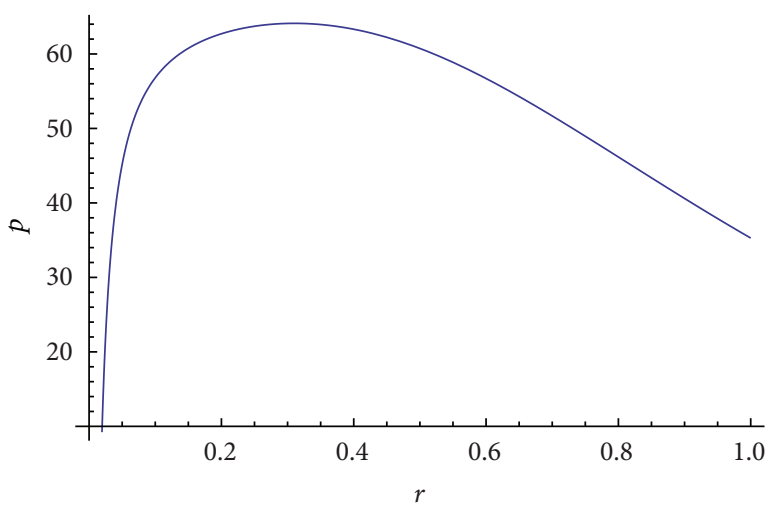

Figure 2: Pressure $p$.

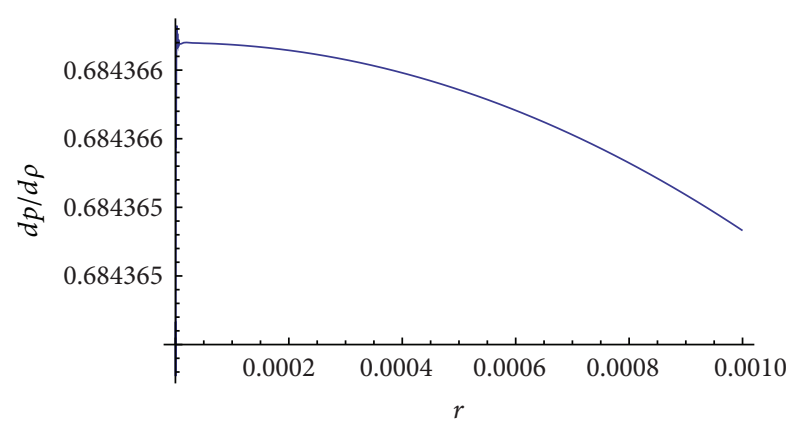

Figure 3: Speed of sound $d p / d \rho$.

\section{Conclusion}

We now comment on the physical properties of the example. We have generated plots for the energy density $\rho$, pressure $p$, and the speed of sound in Figures 1, 2, and 3, respectively. These graphical plots indicate that $\rho$ and $p$ are positive and well behaved. The speed of sound is less than the speed of light as required for causality. Therefore the algorithm presented in this paper produces new solutions which are physically reasonable.

We have generated an algorithm to produce a new solution to the Eistein field equations from a given seed metric. We observe that the resulting model contains isotropic pressures unlike the approach of Chaisi and Maharaj [9] and
Maharaj and Chaisi [10]; in their treatment, the new model has anisotropic pressures. Another advantage of our approach is the use of isotropic coordinates in the formulation of the condition of pressure isotropy. This may leads to new insights into the behaviour of gravity since previous treatments mainly utilised canonical coordinates. The algorithm produced a new solution in terms of integrals containing arbitrary functions. We have shown, with the help of a conformally flat metric, that these integrals may be evaluated in terms of elementary functions. This example suggests that our approach may be extended to other physically relevant metrics.

\section{Acknowledgments}

S. A. Ngubelanga thanks the National Research Foundation and the University of KwaZulu-Natal for financial support. S. D. Maharaj acknowledges that this work is based upon research supported by the South African Research Chair Initiative of the Department of Science and Technology and the National Research Foundation.

\section{References}

[1] M. S. R. Delgaty and K. Lake, "Physical acceptability of isolated, static, spherically symmetric, perfect fluid solutions of Einstein's equations," Computer Physics Communications, vol. 115, no. 2-3, pp. 395-415, 1998.

[2] M. R. Finch and J. E. F. Skea, Unpublished notes, 1998.

[3] H. Stephani, D. Kramer, M. A. H. MacCallum, C. Hoenselaars, and E. Herlt, Exact Solutions of Einstein's Field equations, Cambridge University Press, Cambridge, UK, 2003.

[4] S. Rahman and M. Visser, "Spacetime geometry of static fluid spheres," Classical and Quantum Gravity, vol. 19, no. 5, pp. 935952, 2002.

[5] K. Lake, "All static spherically symmetric perfect-fluid solutions of Einstein's equations," Physical Review D, vol. 67, no. 10, Article ID 104015, 2003.

[6] D. Martin and M. Visser, "Algorithmic construction of static perfect fluid spheres," Physical Review D, vol. 69, no. 10, Article ID 104028, 2004.

[7] P. Boonserm, M. Visser, and S. Weinfurtner, "Generating perfect fluid spheres in general relativity," Physical Review D, vol. 71, no. 12, Article ID 124037, 2005.

[8] L. Herrera, A. Di Prisco, J. Martin, J. Ospino, N. O. Santos, and O. Troconis, "Spherically symmetric dissipative anisotropic fluids: a general study," Physical Review D, vol. 69, no. 8, Article ID 084026, 2004.

[9] M. Chaisi and S. D. Maharaj, "A new algorithm for anisotropic solutions," Pramana, vol. 66, no. 2, pp. 313-324, 2006.

[10] S. D. Maharaj and M. Chaisi, "New anisotropic models from isotropic solutions," Mathematical Methods in the Applied Sciences, vol. 29, no. 1, pp. 67-83, 2006.

[11] P. Kustaanheimo and B. Qvist, "A note on some general solutions of the Einstein field equations in a spherically symmetric world," Societas Scientiarum Fennica, vol. 13, no. 16, p. 12, 1948.

[12] L. Herrera, G. Le Denmat, N. O. Santos, and A. Wang, "Shearfree radiating collapse and conformal flatness," International Journal of Modern Physics D, vol. 13, no. 4, pp. 583-592, 2004. 
[13] S. D. Maharaj and M. Govender, "Radiating collapse with vanishing Weyl stresses," International Journal of Modern Physics D, vol. 14, no. 3-4, pp. 667-676, 2005.

[14] S. S. Misthry, S. D. Maharaj, and P. G. L. Leach, "Nonlinear shear-free radiative collapse," Mathematical Methods in the Applied Sciences, vol. 31, no. 3, pp. 363-374, 2008.

[15] G. Abebe, K. S. Govinder, and S. D. Maharaj, "Lie symmetries for a conformally flat radiating star," International Journal of Theoretical Physics, vol. 52, pp. 3244-3254, 2013. 


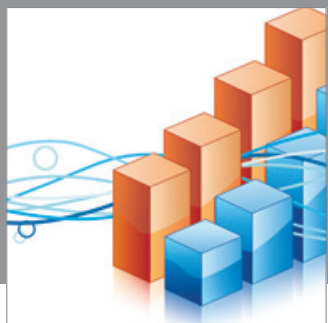

Advances in

Operations Research

mansans

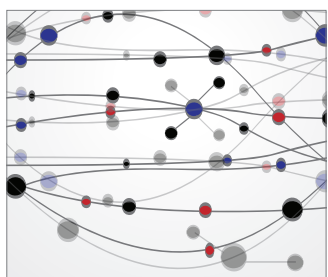

The Scientific World Journal
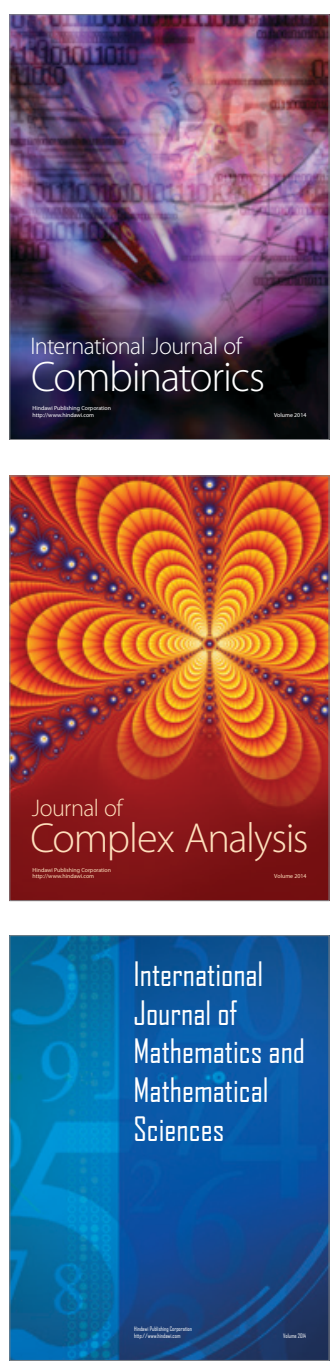
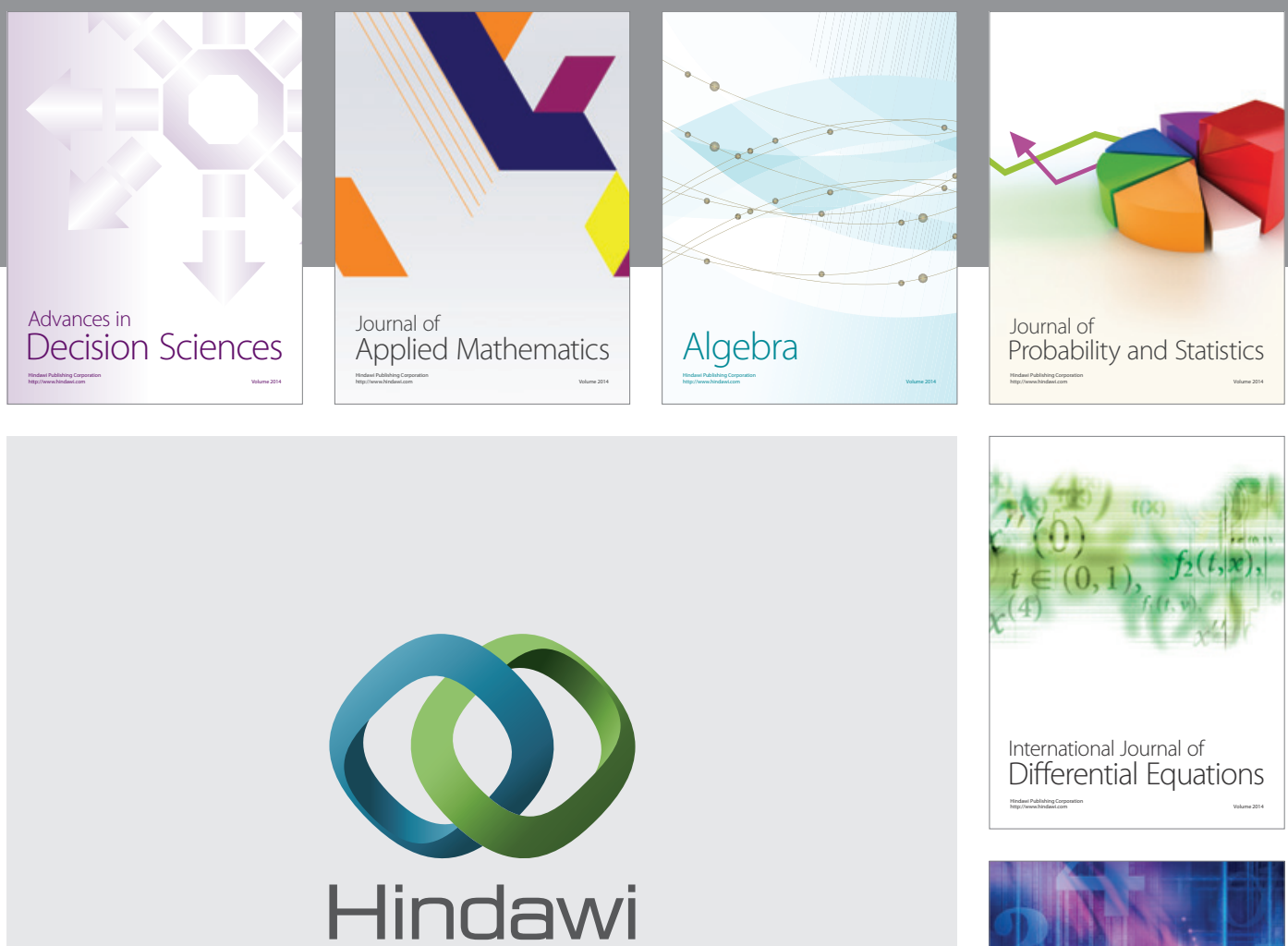

Submit your manuscripts at http://www.hindawi.com
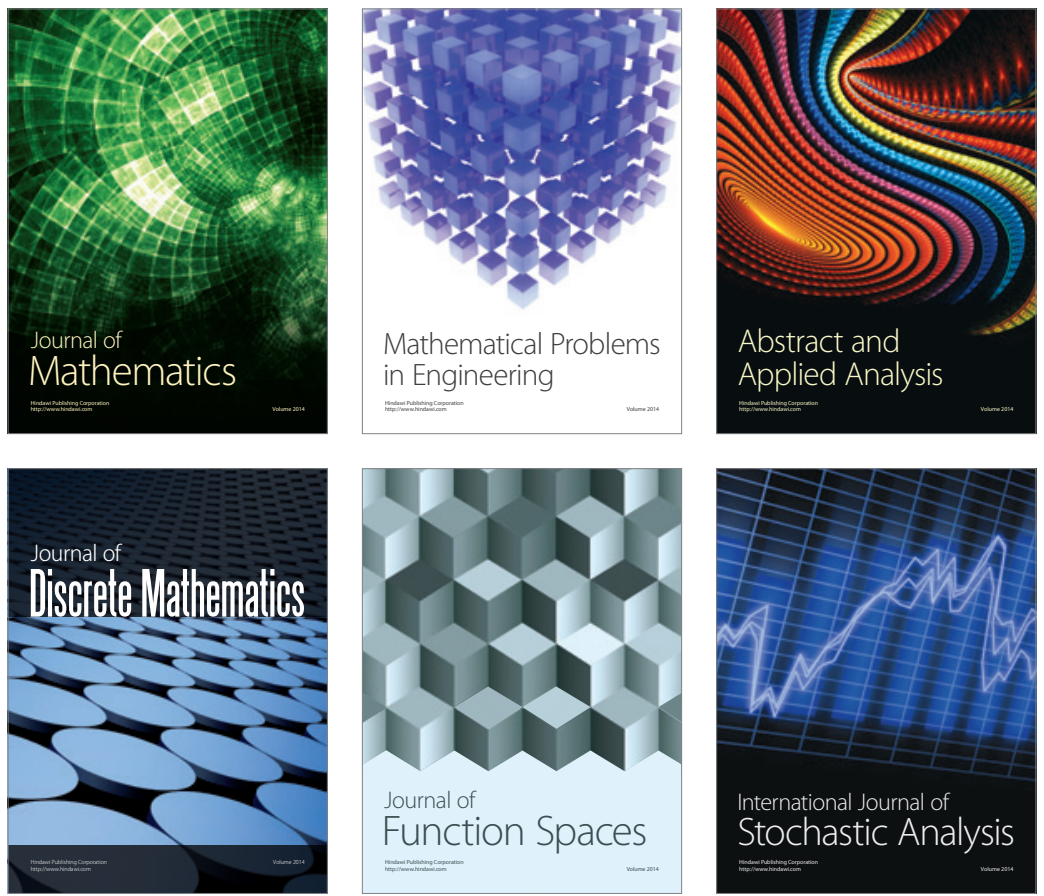

Journal of

Function Spaces

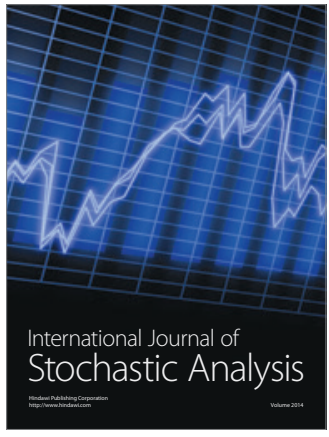

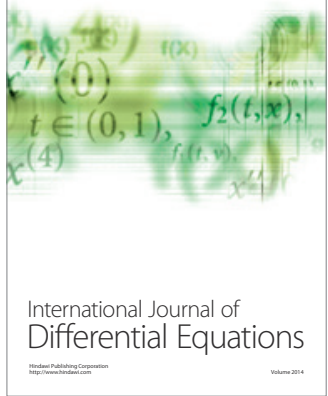
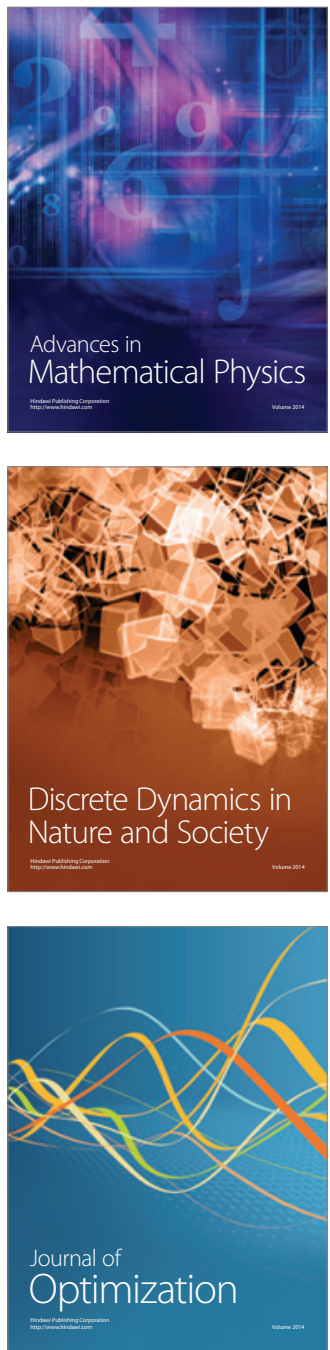